\title{
TUMOR SÓLIDO PSEUDOPAPILAR DEL PÁNCREAS EN LA IV REGIÓN*
}

\author{
Drs. Marcelo A. Beltrán S., ${ }^{1,2}$, Javier Arcos P. ${ }^{2}$, Ramón Hermosilla V. ${ }^{2}$, \\ Oriana Urbina C. ${ }^{2}$, Tatiana Fuentes M. ${ }^{2}$, Paola Andrade L. ${ }^{2}$, Francisco Tello C. ${ }^{2}$ \\ 1 Servicio de Cirugía, Hospital San Juan de Dios de La Serena. \\ 2 Departamento de Clínicas, Facultad de Medicina, Universidad Católica del Norte, IV Región. \\ Chile.
}

\begin{abstract}
Solid-pseudopapillary tumor of pancreas

Introduction: The solid-pseudopapillary tumor of pancreas (STP) is a low-grade malignant neoplasm. In Chile, 21 cases have been reported since 2008, most of them treated in Santiago. The present series contributes to the national case-load and has the purpose to describe the experience with this uncommon neoplasm in the IV Region. Patients and Methods: From January 2004 to March 2014, a total of 38 benign and malignant pancreatic neoplasms have been informed in the data-base of our Regional Pathology Service. We selected the biopsies informed as STP and 5 cases (13\%) were found. The clinical records of these patients were retrospectively reviewed. The results were reported using descriptive statistics with central tendency measures and dispersion. Results: Most patients were women with a mean age of 44.8 years. All patients were studied with either abdominal ultrasound; computed tomography or magnetic resonance. In 2 patients the STP was located in the pancreatic tail, and they were submitted to distal pancreatectomy. The other 3 patients had the STP in the pancreatic head and were resolved by pancreatoduodenectomy. All tumors were confirmed by immunohistochemistry. Late follow-up showed malignant behavior in 1 patient while the other 4 patients are currently free of disease. Conclusions: The general characteristics of STP in the IV Region are similar to what is currently known. They present a high incidence compared with the habitually published incidence.
\end{abstract}

Key words: Pancreatic pseudopapillary tumors, Frantz tumor, pancreas cancer, pancreatectomy.

\section{Resumen}

Introducción: El tumor sólido pseudopapilar del páncreas (TSP) es una neoplasia con bajo potencial maligno. En Chile se han reportado 21 casos desde el año 2008, 18 de ellos tratados en instituciones de Santiago. La presente serie además de contribuir a la casuística nacional, tiene como objetivo la descripción de la experiencia de la IV Región con el manejo de estos tumores. Pacientes y Métodos: Entre enero de 2004 y marzo de 2014 se informaron 38 biopsias de tumores pancreáticos benignos y malignos en la base de datos

*Recibido el 13 de agosto de 2014 y aceptado para publicación el 14 de diciembre de 2014.

Los autores no refieren conflictos de interés.

Correspondencia: Marcelo A. Beltrán S. beltran_01@yahoo.com 
del Servicio de Patología Regional. Se seleccionaron las biopsias informadas como TSP, las que constituyen 5 casos $(13 \%)$ y se revisaron las fichas clínicas en forma retrospectiva. Para el informe de los resultados se utilizó estadística descriptiva con medidas de tendencia central y dispersión. Resultados: El promedio de edad fue 44,8 años, siendo la mayoría mujeres. Los pacientes fueron estudiados con ecografía, tomografía computarizada y resonancia magnética. En 2 pacientes el TSP se localizaba en la cola del páncreas, estos pacientes fueron sometidos a pancreatectomía distal. En los otros 3 casos el TSP se encontraba en la cabeza del páncreas y fueron resueltos mediante pancreatoduodenectomía. Los TSP fueron confirmados por inmunohistoquímica. El comportamiento posterior fue benigno en 4 casos y maligno en 1 caso. Conclusiones: Las características de los TSP en la IV Región son similares a las conocidas y presentan una alta incidencia comparada con la habitualmente reportada.

Palabras clave: Tumor pseudopapilar de páncreas, Tumor de Frantz, Cáncer de páncreas, Pancreatectomía.

\section{Introducción}

El tumor sólido pseudopapilar del páncreas (TSP) es una neoplasia del páncreas exocrino con bajo potencial maligno y crecimiento lento que se presenta típicamente en mujeres jóvenes ${ }^{1-4}$. El TSP fue reportado por primera vez en 1959 por Virginia K. Frantz 5 . En 1970 Hamoudi y colaboradores describieron las características ultraestructurales del TSP lo que llevó a su aceptación como una entidad clínico-patológica diferente ${ }^{3}$. Finalmente el año 1996, fue incluido en la clasificación de tumores de la World Health Organization ${ }^{6}$. Estos tumores representan $1 \%$ a $2 \%$ de todas las neoplasias y $10 \%$ a $15 \%$ de todos los tumores quísticos del páncreas ${ }^{1-4}$. La resección quirúrgica ofrece la posibilidad de una excelente supervivencia a largo plazo $^{7}$, recomendándose el abordaje quirúrgico agresivo en estos pacientes ${ }^{1}$. En Chile se han reportado 21 casos desde el año $2008^{8-13}$, 18 de ellos tratados en instituciones de Santiago ${ }^{8-12}$. Razón por la cual, la presente serie además de contribuir a la casuística nacional, tiene como objetivo la descripción de la experiencia de la IV Región con el manejo de estos tumores durante los últimos 10 años.

\section{Pacientes y Método}

El presente constituye un estudio observacional retrospectivo de una serie de casos consecutivos. Inicialmente se revisó la base de datos del Servicio de Patología Regional buscando todas las biopsias de cirugías pancreáticas realizadas en la IV Región entre el mes de enero de 2004 y marzo de 2014 en los 2 hospitales de mayor complejidad de la región, Hospital San Juan de Dios de La Serena y Hospital San Pablo de Coquimbo. En este período se informaron 38 biopsias de tumores pancreáticos benignos y malignos. Se seleccionaron las biopsias informadas como TSP, las que constituyen un total de 5 casos (13\%). La segunda intervención constituyó la revisión de las fichas clínicas de los pacientes operados por este diagnóstico incluyendo variables clínicas, radiológicas, quirúrgicas, complicaciones, estadía hospitalaria, características de la biopsia, inmunohistoquímica y seguimiento. Para el informe de los resultados se utilizó estadística descriptiva con medidas de tendencia central y dispersión. Se discutieron los hallazgos de la serie y se revisó la literatura actual sobre el tema.

\section{Resultados}

En el período de estudio, se operaron 38 tumores pancreáticos benignos y malignos en la IV Región, la mayor parte de los cuales fueron adenocarcinomas de la cabeza del páncreas (63\%). Los TSP se encontraron con una frecuencia de $13 \%$ del total (Tabla 1). El promedio de edad fue 44,8 años (30 a 60 ), siendo la mayoría mujeres $(80 \%)$. La presentación clínica habitual fue de dolor abdominal inespecífico localizado en el hemiabdomen superior en todos los casos con una evolución variable entre 3 semanas y 5 meses. El estudio radiológico inicial fue una ecografía abdominal que demostró la presencia de una tumoración quística en el páncreas en 4 casos (Figura 1), seguido de una tomografía abdominal computarizada (TC) en 3 pacientes (Figura 2) y una resonancia magnética abdominal (RMA) en 1 caso (Figura 3). En 1 paciente el único estudio fue la TC. Estos estudios permitieron confirmar la presencia de un tumor con características quísticas y sólidas en el páncreas, en 4 casos plantearon dentro de los posibles diagnósticos un TSP. En 2 pacientes el TSP se localizaba en la cola del páncreas, en uno de ellos el diagnóstico inicial fue el de un tumor esplénico con compromiso de la cola del páncreas, estos pacientes fueron sometidos a pancreatectomía distal 
Tabla 1. Tumores de páncreas operados en la IV Región entre enero de 2004 y marzo de 2014

\begin{tabular}{|lrcc}
\hline Sexo & + $24(65 \%) /$ \\
& ô 13 & $(35 \%)$ \\
Edad & $59,4 \pm 12,8$ \\
& $(32-81)$ \\
Diagnóstico & & \\
- Tumor neuroendocrino & 1 & $(3 \%)$ \\
- Adenocarcinoma de papila de Vater & 1 & $(3 \%)$ \\
- Tumor de Frantz (TSP) & $\mathbf{5}$ & $(\mathbf{1 3 \%})$ \\
- Cistoadenoma (mucinoso/seroso) & 7 & $(18 \%)$ \\
- Adenocarcinoma de cabeza de páncreas & 24 & $(63 \%)$ \\
Total & 38 & $(100 \%)$ \\
Localización & & \\
- Cuello & 1 & $(3 \%)$ \\
- Cola & 7 & $(18 \%)$ \\
- Cabeza & 30 & $(79 \%)$ \\
Operación & & \\
- Resección local-Enucleación & 1 & $(3 \%)$ \\
- Pancreatectomía distal & 8 & $(21 \%)$ \\
- Pancreatoduodenectomía & 29 & $(76 \%)$ \\
Hospital & & \\
- Hospital San Juan de Dios de La Serena & 17 & $(45 \%)$ \\
- Hospital San Pablo de Coquimbo & 21 & $(55 \%)$ \\
\hline
\end{tabular}

TSP: Tumor sólido pseudopapilar.

con resección esplénica en bloque. En los otros 3 casos el TSP se encontraba en la cabeza del páncreas $\mathrm{y}$ fueron resueltos mediante una pancreatoduodenectomía. Dos pacientes sufrieron complicaciones, ambos fueron los pacientes con el TSP localizado en la cola del páncreas, uno de ellos presentó una fístula pancreática tipo A y el otro sufrió una perforación inadvertida del colon en el ángulo esplénico requiriendo re-exploración quirúrgica y varios aseos quirúrgicos antes de ser dado de alta. La biopsia permitió la sospecha de TSP por las características

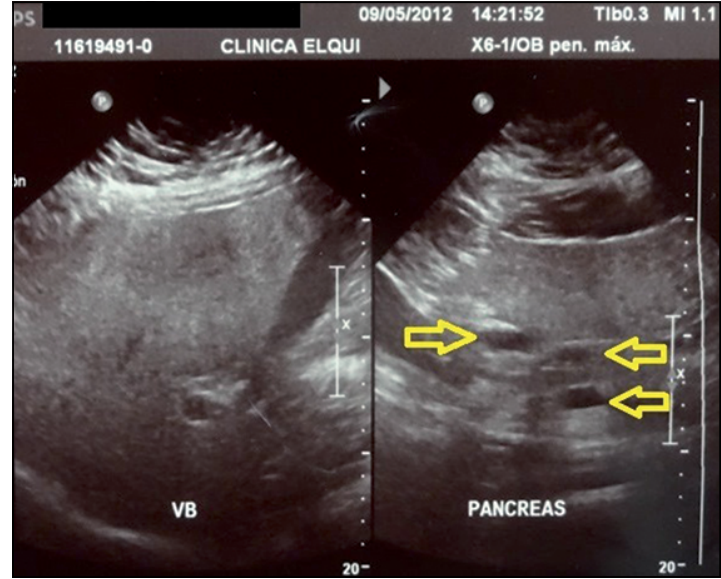

Figura 1. Ecografía abdominal de una paciente de 41 años que muestra imágenes quísticas en la cabeza del páncreas, la paciente continuó su estudio con una resonancia magnética abdominal.

histológicas que en general mostraron tumores bien encapsulados constituidos por células poligonales y degeneración quística sin permeaciones vasculares o linfáticas. En el único paciente masculino de la serie con un tumor de la cola del páncreas, el TSP presentaba invasión macroscópica del bazo y microscópica del parénquima pancreático adyacente con permeaciones linfáticas y vasculares, los que constituyen signos de comportamiento maligno. El TSP fue confirmado por inmunohistoquímica en todos los casos. El comportamiento posterior de los TSP fue benigno en 4 casos. En el TSP con características malignas, el seguimiento demostró recurrencia del tumor a los 3 años con metástasis hepáticas y peritoneales lo que llevó al fallecimiento del paciente. Las otras pacientes se encuentran asintomáticas hasta el presente. Las características generales de los TSP operados en la IV Región se resumen en la Tabla 2. En la Tabla 3
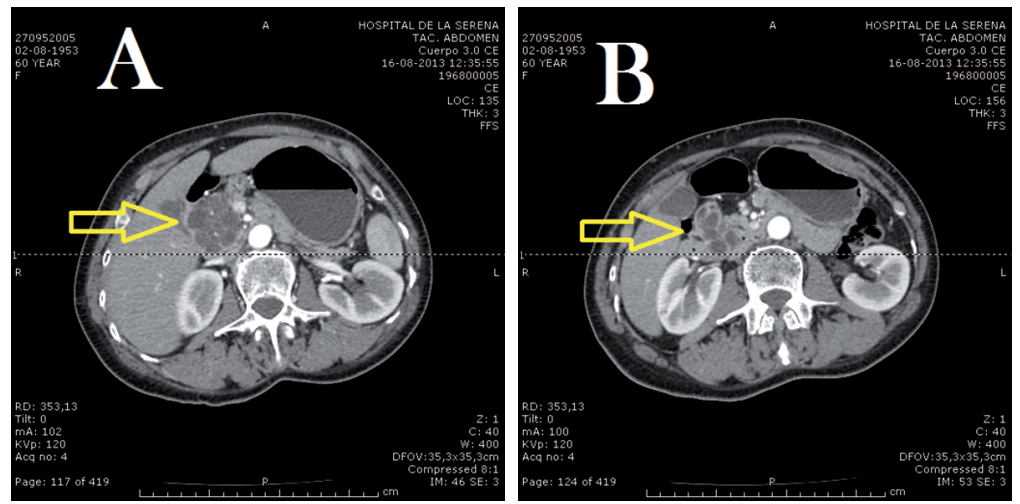

Figura 2. A) Tomografía computarizada abdominal en una paciente de 60 años de edad que muestra un tumor pseudopapilar de la cabeza del páncreas, el corte muestra el componente principalmente quístico del tumor; B) Corte transversal que muestra el tumor pseudopapilar con su componente sólido, el tumor medía aproximadamente $5 \mathrm{~cm}$ de diámetro. La paciente fue resuelta mediante una pancreatoduodenectomía. 

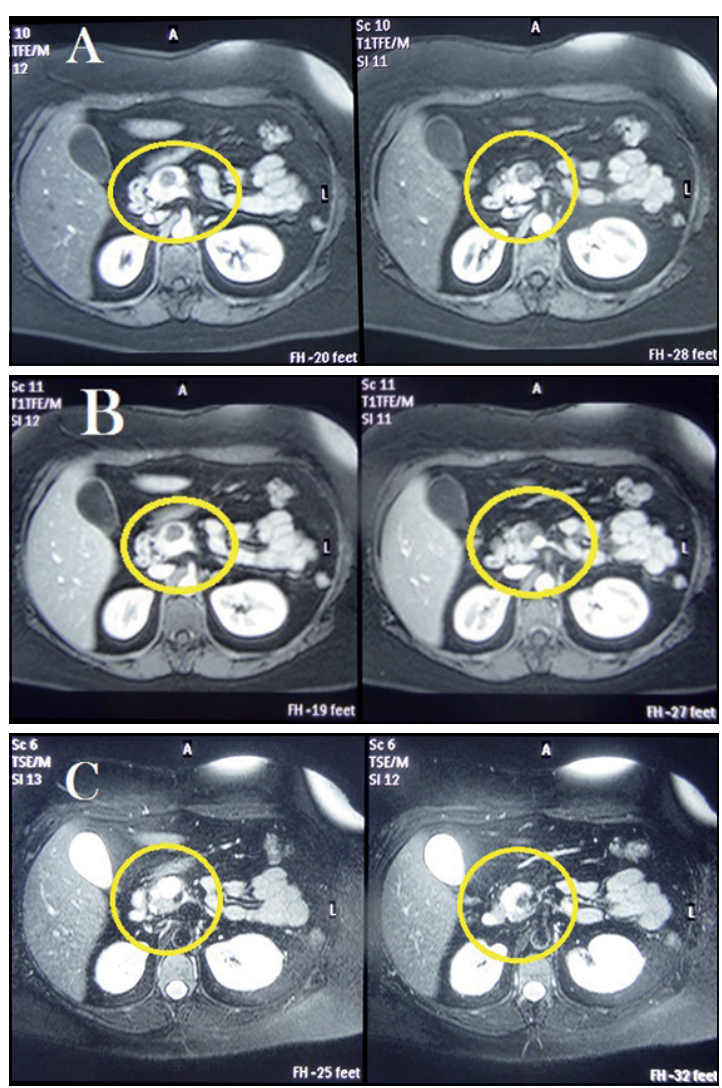

Figura 3. A) Fase arterial T1 de una resonancia magnética abdominal en una paciente de 41 años con un TSP de cabeza de páncreas, destaca el componente sólido del tumor; B) La fase portal T1 no demuestra mayor intensificación del TSP; C) Imagen T2 que muestra mayor intensidad del componente sólido del TSP. La paciente fue sometida a pancreatoduodenectomía. se muestran las características generales de los TSP operados en Chile y reportados en los últimos años. Se puede observar que los TSP en la IV Región se presentaron en pacientes con un promedio de edad mayor al reportado y un paciente fue masculino con un TSP maligno. La localización de los tumores fue la habitual así como las operaciones utilizadas para resolverlos.

\section{Discusión}

El TSP se presenta típicamente en pacientes femeninos jóvenes ${ }^{1-22}$. El TSP en pacientes masculinos es menos frecuente y el promedio de edad en estos casos es mayor, alrededor de los 50 años ${ }^{1,16}$, esto sugiere que el desarrollo del TSP en pacientes masculinos podría ser un evento más tardío y probablemente debido a un crecimiento más lento ${ }^{1}$. En nuestra serie predominaron las mujeres con un promedio de edad similar al promedio habitualmente reportado que varía entre 13 y 45 años en las series internacionales $^{1-4,14-19}$ y entre 6 y 28 años en las publicaciones nacionales ${ }^{8-13}$. Se ha postulado que el TSP se origina en células acinares primitivas ${ }^{3}$, aunque la patogénesis del TSP es desconocida ${ }^{2,3}$. La tendencia a presentarse en pacientes femeninos jóvenes ha llevado a la sugerencia no comprobada de que las hormonas sexuales podrían tener algún $\mathrm{rol}^{2,9,15}$. El TSP constituye una neoplasia atípica debido a su comportamiento indolente habitualmente benigno pero con potencial maligno hasta en $15 \%$ de los $\operatorname{casos}^{15}$. Representa menos de $2 \%$ de todos los tumores pancreáticos y $10 \%$ a $15 \%$ de los tumores quísticos ${ }^{1-20}$. En la IV Región el TSP se presentó en

Tabla 2. Características de los tumores de Frantz operados en la IV Región

\begin{tabular}{|c|c|c|c|c|c|c|c|}
\hline Sexo & Edad & Radiología & Cirugía & $\begin{array}{l}\text { Estadía } \\
\text { (días) }\end{array}$ & Complicaciones & $\begin{array}{c}\text { Inmunohistoquímica } \\
\text { positiva }\end{array}$ & Evolución \\
\hline q & 42 & $\begin{array}{l}\text { Ecografía } \\
\text { TC* }\end{array}$ & $\begin{array}{l}\text { Pancreatectomía distal + } \\
\text { Esplenectomía }\end{array}$ & 47 & $\begin{array}{l}\text { Perforación de } \\
\text { colon }\end{array}$ & $\begin{array}{c}\text { Vimentina } \\
\text { Alfa } 1 \text { Antitripsina }\end{array}$ & $\begin{array}{l}\text { Viva: } \\
8 \text { años }\end{array}$ \\
\hline$\sigma^{\pi}$ & 51 & $\mathrm{TC}$ & $\begin{array}{l}\text { Pancreatectomía distal + } \\
\text { Esplenectomía }\end{array}$ & 17 & $\begin{array}{c}\text { Fístula pancreática } \\
\text { tipo A }\end{array}$ & $\begin{array}{c}\text { CD10-CD56 } \\
\text { Vimentina } \\
\text { Alfa } 1 \text { Antitripsina }\end{array}$ & $\begin{array}{l}\text { Fallece: } \\
3 \text { años }\end{array}$ \\
\hline q & 30 & $\begin{array}{l}\text { Ecografía } \\
\text { TC }\end{array}$ & Pancreatoduodenectomía & 8 & No & $\begin{array}{c}\text { CD10-CD56 } \\
\text { Vimentina } \\
\text { Alfa } 1 \text { Antitripsina }\end{array}$ & $\begin{array}{l}\text { Viva: } \\
6 \text { años }\end{array}$ \\
\hline 우 & 41 & $\begin{array}{l}\text { Ecografía } \\
\text { RMA** }^{* *}\end{array}$ & Pancreatoduodenectomía & 8 & No & $\begin{array}{c}\text { CD10-CD56 } \\
\text { Vimentina }\end{array}$ & $\begin{array}{l}\text { Viva: } \\
2 \text { años }\end{array}$ \\
\hline q & 60 & $\begin{array}{c}\text { Ecografía } \\
\text { TC }\end{array}$ & Pancreatoduodenectomía & 6 & No & $\begin{array}{l}\text { CD10-CD56 } \\
\text { Vimentina }\end{array}$ & $\begin{array}{l}\text { Viva: } \\
7 \text { meses }\end{array}$ \\
\hline
\end{tabular}

*TAC: Tomografía abdominal computarizada. **RMA: Resonancia magnética abdominal. 
Tabla 3. Tumores de Frantz operados en Chile (2008-2014)

\begin{tabular}{|c|c|c|c|c|c|c|c|c|}
\hline Autor & Año & Casos & $\begin{array}{l}\text { Edad } \\
\text { (años) }\end{array}$ & Sexo & $\begin{array}{l}\text { n de } \\
\text { tumores }\end{array}$ & Localización & $\begin{array}{c}\text { Tamaño } \\
\text { tumoral } \\
(\mathrm{cm})\end{array}$ & Operación \\
\hline Acuña R. $^{8}$ & 2008 & 1 & 6 & O: 1 & 2 & $\begin{array}{l}\text { Cabeza: } 1 \\
\text { Cola: } 1\end{array}$ & $2-15$ & $\begin{array}{l}\text { Enucleación: } 1 \\
\text { Pancreatectomía distal: } 1\end{array}$ \\
\hline De Aretxabala X. ${ }^{9}$ & 2008 & 1 & 15 & ㅇ: 1 & 1 & Cabeza: 1 & 8 & Pancreatoduodenectomía: 1 \\
\hline Venturelli M. ${ }^{13}$ & 2010 & 3 & $22-42$ & q: 3 & 3 & $\begin{array}{l}\text { Cuerpo: } 1 \\
\text { Cola: } 2\end{array}$ & $3-12$ & Pancreatectomía distal: 3 \\
\hline Zúñiga JM. ${ }^{11}$ & 2011 & 1 & 32 & ㅇ: 1 & 2 & $\begin{array}{l}\text { Cabeza: } 1 \\
\text { Cola: } 1\end{array}$ & $6-8$ & $\begin{array}{l}\text { Pancreatoduodenectomía: } 1 \\
\text { Pancreatectomía distal: } 1\end{array}$ \\
\hline Carreño L. ${ }^{12 *}$ & 2011 & $5^{*}$ & $13-27$ & ㅇ: 5 & 5 & $\begin{array}{l}\text { Cabeza: } 1 \\
\text { Cuerpo: } 2 \\
\text { Cola: } 2\end{array}$ & $4-10$ & $\begin{array}{l}\text { Pancreatoduodenectomía: } 1 \\
\text { Pancreatectomía distal: } 4\end{array}$ \\
\hline Slako M. ${ }^{10}$ & 2012 & 11 & $14-43$ & $\begin{array}{l}\text { o. } 10 \\
\text { त̂t: } 1\end{array}$ & 11 & $\begin{array}{l}\text { Cabeza: } 2 \\
\text { Cuerpo: } 5 \\
\text { Cola: } 4\end{array}$ & $2-13$ & $\begin{array}{l}\text { Pancreatoduodenectomía: } 2 \\
\text { Pancreatectomía distal: } 9\end{array}$ \\
\hline Beltrán M.** & 2014 & 5 & $30-60$ & $\begin{array}{l}\text { o: } \\
\text { ô: } 1\end{array}$ & 5 & $\begin{array}{l}\text { Cabeza: } 3 \\
\text { Cola: } 2\end{array}$ & $3-12$ & $\begin{array}{l}\text { Pancreatoduodenectomía: } 3 \\
\text { Pancreatectomía distal: } 2\end{array}$ \\
\hline Total & & 26 & $\begin{array}{c}29 \\
(6-60)\end{array}$ & $\begin{array}{l}\text { o: } 24 \\
\text { ô: } 2\end{array}$ & 29 & $\begin{array}{l}\text { Cabeza: } 9(32 \%) \\
\text { Cuerpo: } 8(28 \%) \\
\text { Cola: } 12(42 \%)\end{array}$ & $2-15$ & $\begin{array}{l}\text { Enucleación: } 1(4 \%) \\
\text { Pancreatoduodenectomía: } \\
8(28 \%) \\
\text { Pancreatectomía distal: } \\
20(68 \%)\end{array}$ \\
\hline
\end{tabular}

*Un tumor previamente reportado9. **Serie actual.

$13 \%$ de todos los pacientes sometidos a resecciones pancreáticas tumorales, esta alta incidencia se debe seguramente a un sesgo ocasionado por el pequeño número de pancreatectomías realizadas.

La presentación clínica de los TSP es inespecífica por lo que el diagnóstico es muchas veces incidental durante estudios radiológicos por otras causas ${ }^{2}$. Habitualmente se presentan con dolor abdominal o dorsal $(11 \% \text { a } 67 \%)^{1-24}$, distensión abdominal $(16 \%$ a $27 \%)^{2,4}$, náusea y vómito $(9 \%)^{2}$, masa abdominal palpable $(15 \%)^{4,14}$, pérdida de peso, y ocasionalmente ictericia $(1 \%)^{3,4}$. Sin embargo, pueden ser asintomáticos entre $28 \%$ y $80 \%$ de los $\operatorname{casos}^{1,3,4,16,18}$. Los síntomas se deben y dependen del desarrollo del tumor y del efecto de masa que este produce ${ }^{16}$. El tiempo de duración de los síntomas no es muy prolongado, habitualmente es menor a 3 meses $^{2}$, en otros casos puede ser superior a 6 años ${ }^{15}$. Nuestros pacientes se presentaron todos ellos con dolor abdominal inespecífico de corta evolución concordando con los reportes publicados. El diagnóstico preoperatorio de TSP es difícil, y la exactitud diagnóstica insuficiente reportándose entre $19 \%$ y $82 \%$ en diferentes series ${ }^{1,2}$. Una de las razones, es la variedad de imágenes que presenta el TSP, las que varían entre tumores completamente sólidos a tumores completamente quísticos ${ }^{1,2,10}$. Estas observaciones sugieren que el TSP inicialmente constituye un tumor sólido y que al crecer degenera volviéndose quístico debido a necrosis y hemorragia intratumoral, por lo que los hallazgos radiológicos dependen del tamaño tumoral $^{1}$. El estudio inicial en estos pacientes consiste habitualmente en una ecografía abdominal, la que reporta una tumoración sólida quística encapsulada sin dilatación del conducto pancreático principal en la cabeza, cuerpo o cola del páncreas ${ }^{4,17}$. Frecuentemente el siguiente estudio es la TC, que permite una excelente visualización de las características del tumor y su localización anatómica y topográfica. La presentación tomográfica del TSP es la de una masa compleja, encapsulada, con un componente sólido y otro quístico que se realzan en la fase arterial y en la fase venosa portal $1^{1,2,4,12,15,17}$. Los tumores mayores a $3,5 \mathrm{~cm}$ presentan estas características clásicas, $\sin$ embargo, los tumores menores a $3 \mathrm{~cm}$ se presentan como masas sólidas ${ }^{1,4}$. La dilatación de la vía biliar debida al TSP no es un hallazgo frecuente ${ }^{17}$. La presencia de metástasis y compromiso vascular en la TC señalan un tumor maligno ${ }^{1}$. Otro estudio disponible es la RMA, la que diferencia mejor que 
la TC los componentes sólidos y quísticos del TSP, demostrando las áreas hemorrágicas ${ }^{17} \mathrm{y}$ proveyendo mayor información respecto a la resecabilidad ${ }^{2-4}$. Los TSP en RMA presentan elevada intensidad de señal del componente sólido en imágenes T1 y elevada intensidad del componente quístico en imágenes $\mathrm{T} 2^{14,17}$. Algunos autores han reportado el uso de tomografía de emisión de positrones (PET) con excelentes resultados para el diagnóstico específico de TSP ${ }^{1,4,16}$. El rol de la endosonografía asociado a punción con aguja fina y citopatología se ha establecido para la confirmación del diagnóstico de TSP en casos de tumores pequeños menores a $3 \mathrm{~cm}$ y para descartar otros tumores pancreáticos como tumores neuroendocrinos y carcinomas de células acinares en el caso de tumores mayores a $3 \mathrm{~cm}$, con una precisión que alcanza hasta $100 \%^{1-3,14,21}$. Se han reportado complicaciones con este procedimiento como la siembra tumoral en el trayecto de la aguja, hemorragia y fístulas pancreáticas y biliares ${ }^{2}$. En nuestra región la endosonografía no es un estudio disponible. En nuestra serie, 4 pacientes se estudiaron inicialmente con una ecografía la que demostró la tumoración pancreática, en 3 casos se continuó el estudio con una TC y en 1 caso con una RMA, un paciente fue estudiado inicialmente con una TC.

El tratamiento del TSP es la completa y agresiva resección quirúrgica, aun en el caso de tumores localmente invasores y en el caso de tumores diseminados con metástasis a distancia ${ }^{1-4,14}$. El uso de quimioterapia y radioterapia ha sido anecdóticamente reportado pero no tienen un rol en el tratamiento de estos tumores ${ }^{3}$. El tipo de resección depende de la localización del tumor, la localización más frecuente es la cola del páncreas (33\% a $80 \%)$, seguida por la cabeza (32\% a $67 \%)$, cuerpo $(14 \%$ a $27 \%$ ) y proceso uncinado $(3 \%)^{3,4,14,18}$. Habitualmente se realizan pancreatectomías distales para los tumores de cuerpo y cola y pancreatoduodenectomías para los tumores de cabeza y cuello $1,4,10,12,19$. Aunque la enucleación de tumores pequeños ha sido descrita, esta no se recomienda debido a que los TSP presentan un patrón de crecimiento celular infiltrante al parénquima pancreático adyacente y a los tejidos circundantes ${ }^{1}$, tal y como se presentó en un paciente de esta serie. La linfadenectomía no está habitualmente indicada en TSP localizados debido a que las metástasis linfáticas son extremadamente infrecuentes en estos $\operatorname{casos}^{1,2,16}$. El abordaje quirúrgico de los TSP es tradicionalmente la cirugía abierta. Se ha descrito el abordaje laparoscópico para TSP menores de 3 $\mathrm{cm}$ de la cola del páncreas con buenos resultados quirúrgicos, baja morbilidad y buen pronóstico a largo plazo por lo que se surgiere la pancreatectomía laparoscópica como técnica de elección para los TSP de cuerpo y cola ${ }^{2,4,19,20}$. Las complicaciones de la cirugía pancreática por TSP son las que habitualmente se presentan con las intervenciones quirúrgicas en el páncreas por otras causas y consisten principalmente en fístulas, pseudoquistes e infartos esplénicos $2,4,10,16,19$. Dos de nuestros pacientes sufrieron complicaciones, una relacionada directamente con la cirugía como es la perforación del colon y la otra esperable en resecciones de la cola del páncreas como es la fístula pancreática.

El TSP es un tumor bien encapsulado ${ }^{22}$, aun así la interface microscópica entre el tumor y el parénquima pancreático adyacente muestra un patrón infiltrante de crecimiento limitado hasta en $90 \%$ de todos los $\operatorname{casos}^{1,14}$. El TSP al corte presenta áreas sólidas, quísticas, pseudopapilares y en algunos casos hemorrágicas en diversas proporciones ${ }^{2,4,12,14,22}$. Histológicamente el TSP se caracteriza por áreas sólidas que alternan con áreas pseudopapilares y espacios quísticos ${ }^{22}$. Las áreas sólidas se componen de cordones de células medianas poligonales uniformes sin aumento de atipias citológicas y escasas mitosis, en un estroma muy vascularizado rodeado de una cápsula fibrosa $a^{2-4,15,21,22}$. Los criterios de malignidad para TSP son la invasión de la cápsula pancreática, diseminación tumoral en el parénquima pancreático, invasión de los tejidos adyacentes al páncreas, la presencia de permeaciones vasculares y perineurales y un Ki-67 mayor a 5\% ${ }^{1-3,22}$. Los marcadores inmunohistoquímicos habitualmente utilizados en el TSP son Vimentina, Alfa-1-Antitripsina, Enolasa Neuronal Específica, CD10 y CD56 $6^{2,412,14,15,22}$. En algunos casos se han medido receptores hormonales para progesterona y estrógenos los que se encuentran ocasionalmente presentes ${ }^{2,22}$. En algunos tumores es posible encontrar la expresión focal de Sinaptofisina, Citokeratina y Cromogranina- ${ }^{2}$. El $\mathrm{Ki}-67$ se encuentra elevado en TSP malignos ${ }^{2}$. En nuestros pacientes los marcadores utilizados fueron CD10, CD56, Alfa-1-Antitripsina y Vimentina los que fueron positivos.

El pronóstico del TSP es bueno a largo plazo lográndose una excelente sobrevivencia alejada a 5 años o más en más de $95 \%$ de los pacientes ope$\operatorname{rados}^{2-4,16}$, aún en tumores metastásicos ${ }^{1}$. Por esta razón la recomendación actual es la cirugía resectiva agresiva $^{1-3}$. Más de $95 \%$ de todos los pacientes con TSP localizados en el páncreas son curados con la resección completa del tumor ${ }^{2}$. La recurrencia local se presenta en menos de $10 \%$ de los casos dentro de los primeros 4 años después de la cirugía ${ }^{2,14}$. El TSP se comporta como un tumor relativamente benigno con un bajo índice de malignidad, la incidencia de degeneración maligna ha sido reportada por debajo de $15 \%$. ${ }^{1,3}$. Se ha sugerido que algunos factores como el sexo masculino, pacientes jóvenes, diámetro tumoral mayor a $5 \mathrm{~cm}$, invasión venosa, necrosis 
tumoral, patrón de crecimiento invasor y un Ki-67 elevado se asocian a degeneración maligna del tumor $^{7}$, otros estudios no han demostrado asociación con estos factores ${ }^{1,2}$. Los únicos factores estadísticamente comprobados que aumentan el riesgo de malignidad son un diámetro tumoral mayor a $6 \mathrm{~cm}$ y un Ki-67 elevado ${ }^{1-3}$. En nuestros casos, 4 pacientes tenían TSP menores a 6 de diámetro y 1 paciente de sexo masculino tenía un TSP de aproximadamente 12 a $13 \mathrm{~cm}$ de diámetro, este paciente falleció por enfermedad metastásica.

\section{Conclusiones}

Las características de los TSP en la IV Región son similares a las conocidas y presentan una alta incidencia comparada con lo habitualmente reportado, este hecho se debe seguramente a un sesgo secundario al número de resecciones pancreáticas consideradas factibles para tumores pancreáticos en nuestra Región.

\section{Referencias}

1. Hosokawa I, Shimizu H, Ohtsuka M, Kato A, Yoshitomi H, Furukawa K, et al. Preoperative diagnosis and surgical management for solid pseudopapillary neoplasm of the pancreas. J Hepatobiliary Pancreat Sci. 2014;21:573-8.

2. Wan XG, Ni QF, Fei JG, Zhong ZX, Yu PF. Clinicopathological features and surgical outcome of solid pseudopapillary tumor of the pancreas: Analysis of 17 cases. World J Surg Oncol. 2013;11:38.

3. Vassos N, Agaimy A, Klein P, Hohenberger W, Croner RS. Solid-pseudopapillary neoplasm (SPN) of the pancreas: Case series and literature review on an enigmatic entity. Int J Clin Exp Pathol. 2013;6:1051-9.

4. Cai Y, Ran X, Xie S, Wang X, Peng B, Mai G, et al. Surgical management and long-term follow-up of solid pseudopapillary tumor of the pancreas: A large series from a single institution. J Gastrointest Surg. 2014;18:935-40.

5. Frantz VK. Tumors of the pancreas. In: Frantz VK (ed). Atlas of tumor pathology. Washington, DC: US Armed Forces Institute of Pathology; 1959. p:32-3.

6. Kloppel G, Hruban RH, Klimstra DS, Maitra A, Morohoshi T, Notohara K, et al. Solid-pseudopapillary neoplasm of the pancreas. In: Bosman FT, Carneiro F, Hruban RH, Theise ND (eds). WHO classification of tumors of the digestive system. Lyon: IARC; 2010. $\mathrm{p}: 327-30$.

7. Tipton SG, Smyrk TC, Sarr MG, Thompson GB. Malignant potential of solid pseudopapillary neoplasm of the pancreas. Br J Surg. 2006;93:733-7.

8. Acuña R, Soto $P$, Muñoz V, Acuña J. Tumor sólido pseudopapilar de páncreas, reporte de un caso. Rev Chil Pediatr. 2008;79:404-8.

9. De Arextabala X, Rencoret G, Maluenda G, Fernández C, Csendes A. Tumor sólido pseudopapilar del páncreas: Caso Clínico. Rev Chil Cir. 2008;60:241-5.

10. Slako M, Muñoz C, Briceño E, Moisan F, Martínez J, Patillo JC, y cols. Tumor sólido pseudopapilar del páncreas: Estudio descriptivo de una serie de casos. Rev Chil Cir. 2012;64:251-6.

11. Zúñiga JM, Buchheister M, Valdés F. Doble tumor sólido pseudopapilar del páncreas. Rev Chil Cir. 2011;63:13-4.

12. Carreño L, Smok G, Villarroel MA, Sanhueza V. Neoplasia sólida seudopapilar del páncreas: Comunicación de 5 casos. Gastroenterol Hepatol. 2011;34:266-70.

13. Venturelli F, Carrasco C, del Pozo M, Cárcamo C, Cárcamo M, Venturelli A. Tumor sólido-pseudopapilar del páncreas. Presentación de 3 casos clínicos. Cuad Cir. 2010;24:11-6.

14. Patil TB, Shrikhande SV, Kanhere HA, Saoji RR, Ramadwar MR, Shukla PJ. Solid pseudopapillary neoplasm of the pancreas: A single institution experience of 14 cases. HPB 2006;8:148-50.

15. Miglani RK, Nagesh NS, Murthy DS, Bhat RS, Nayak RS, Ashok KK. Solid pseudopapillary tumor of pancreas: A rare neoplasm. Indian J Surg. 2010;72:158-60.

16. Igbinosa O. Pseudopapillary tumor of the pancreas: An algorithmic approach. J Pancreas 2011;12:262-5.

17. Dong DJ, Zhang SZ. Solid-pseudopapillary tumor of the pancreas: CT and MRI features of 3 cases. Hepatobiliary Pancreat Dis Int. 2006;5:300-4.

18. Coelho JC, Valle CL, Ribas BM, Andriguetto LD, Claus CM. Surgical treatment of cystic neoplasm of the pancreas. Arq Gastroenterol. 2010;47:135-40.

19. Jarry J, Bodin R, Peycru T, Núñez M, Collet D, Sa Cunha A. Role of laparoscopic distal pancreatectomy for solid pseudopapillary tumor. J Soc Laparoendoscopic Surg. 2012;16:552-8.

20. Zhang RC, Yan JF, Xu XW, Chen K, Ajoodhea H, Mou YP. Laparoscopic vs open distal pancreatectomy for solid pseudopapillary tumor of the pancreas. World J Gastroenterol. 2013;19:6272-7.

21. Mehta N, Modi L, Patel T, Shah M. Study of Cytomorphology of solid pseudopapillary tumor of the pancreas and its differential diagnosis. J Cytol. 2010;27:118-22.

22. Santini D, Poli F, Lega S. Solid-papillary tumors of the pancreas: Histopahology. J Pancreas 2006;7:131-6. 